\title{
Shelf Space Allocation and Green Technology R\&D in Green Agricultural Products Supply Chain
}

\author{
Guohua Sun ${ }^{\mathrm{a}}$, Caihong Xub
}

School of Management Science and Engineering, Shandong University of Finance and

Economics, Jinan 250014, China.

anksungh@qq.com, ${ }^{\mathrm{b}} 15806678863 @ 163 . c o m$

Keywords: agricultural products supply chain; shelf space; coordination contract

Abstract: This paper examines the shelf space allocation and R\&D of green technology in the green agricultural products supply chain(GAPSC) with one supplier and one retailer. The retail demand is influenced by the green level and shelf space allocated. The optimal equilibriums are investigated in the GAPSC with unequal channel powers, namely supplier-Stackelberg and retailer-Stackelberg. The results show that in both GAPSCs, the supplier makes effort in R\&D of green technology only when the consumers are sensitive to the green level and insensitive to the retail price, the marginal cost related to the improvement of green level increases slowly.

\section{Introduction}

With the increasing growth of population and deterioration of environment, agriculture production is required not only to yield more outputs, but also to be environmentally sustainable. The problems of soil degradation, water pollution and loss of biodiversity caused by the environmentally inappropriate practices have become the public focus(Ning et al. 2017). GAPSC taking environment influence and resource consumption into consideration is considered as an effective way to promote the production of agricultural products less environmentally disruptive.

The sales of agricultural products are affected by the shelf space allocated. It's estimated that the average shelf space elasticity is 0.17 (Eisend, 2014). That is, the sales quantity of agricultural products will increase 17\%, as the shelf space allocated is doubled. Different form the general agricultural products, the green agricultural products are usually packaged and displayed one by one on the shelf in most supermarkets. The sales of green agricultural products are not as fantastic as the general agricultural products allocated more shelf spaces which spoil the GAPSC members' initiatives to a certain degree.

There is a lot of work done on the shelf space allocation. Lu and Zhu(2010) consider a supply chain with two competing manufacturers and a common retailer in which the market demand of each product is affected by the product quality and the shelf space allocated. Luo et al.(2010) coordinated the supply chain with multiple heterogeneous retailers via wholesale price and holding cost subsidy. Zhou et al.(2012) considered a supply chain with inventory-dependent demand, in which the trade credit is offered by the supplier. Tsao et al. (2014) developed a multi-player retailer-Stackelberg game to study the interaction between the retailer and manufacturers. Amita et al.(2015) developed an optimal shelf-space stocking policy when demand is inventory-dependent in addition to the exogenous uncertainty. Hübner and Schaal(2017) investigated the shelf-space planning problem with stochastic demand sensitive to the number and position of facings.

The green supply chain has been a hot topic for several decades. Ghosh and Shah (2012) studied the effect of decentralized decision-making and negotiation while bargaining on the greening level of products. Huang et al.(2016) studied the effects of production line design, supplier selection, and choice of transport strategies on profit and control of greenhouse gas emissions. Jamali and Rasti-Barzoki(2018) investigated the pricing and determination of the green degree of a product in competition with a non-green product. Song and Gao(2018) studied the retailer-led revenue-sharing contract and bargaining revenue-sharing contract in the green supply chain.

The existing papers didn't consider the effect of shelf space on GAPSC. Whether the green level of green agricultural products can be increased by allocating more shelf spaces was not studied. In this paper how to allocate shelf space more reasonably is studied by analyzing the optimal decisions of the members in the GAPSCs with unequal channel powers. 


\section{Model Description}

The GAPSC is composed of one supplier and one retailer. The supplier is in charge of producing and $\mathrm{R} \& \mathrm{D}$ of green technology. The retailer is in charge of selling green agricultural products by displaying them on the shelf space. The retail demand is affected by retail price $p$, green level $g$ and shelf space $S$ allocated. That is, $D(p, g, S)=\alpha-\beta p+\gamma g+\delta S$, in which $\alpha$ is the basic demand, $\beta, \gamma, \delta(\geqslant 0)$ are the elasticities of retail price, green level and shelf space allocated.

The unit production cost incurred by the supplier is $c+\rho g$ (Banker et al, 1998), in which $c$ is the current production cost when the supplier doesn't make any effort in R\&D of green technology and $\rho(\geqslant 0)$ denotes the marginal cost as the green level is improved. When the green level is increased to $g$, the investment cost of $\mathrm{R} \& \mathrm{D}$ incurred by the supplier is $\mu g^{2} / 2$. For the retailer, when the shelf space allocated to the agricultural products is $S$, the cost incurred is $\lambda S^{2} / 2$.

\section{Model Analysis}

The optimal equilibriums in the decentralized and centralized GAPSC are analyzed in this section. In the decentralized GAPSC, there is no coordination between the supplier and the retailer. They make decisions independently aiming to maximize their own profits. Let the subscript $i=s, r$ denotes the supplier and retailer, respectively. The optimization problems of the supplier and retailer can be expressed as follows:

$$
\begin{aligned}
& \max \pi_{s}(\omega, g)=(\omega-c-\rho g)(\alpha-\beta p+\gamma g+\delta S)-\frac{\mu}{2} g^{2} \\
& \max \pi_{r}(p, S)=(p-\omega)(\alpha-\beta p+\gamma g+\delta S)-\frac{\lambda}{2} S^{2}
\end{aligned}
$$

The supplier and the retailer usually have different channel powers. The member having more power is the Stackelberg leader and makes decisions first. The other member will make his choices according to the leader's decisions. The sequence of events in GAPSC can be described as below.

Model $S$ : The supplier first sets the wholesale price $\omega$ and the green level $g$. Then, the retailer determines the retail price $p$ and the shelf space $S$ allocated to the agricultural products after observing the supplier's decisions.

Model $R$ : The retailer first sets the marginal price $m(=p-\omega)$ and allocates shelf space $S$ to the agricultural products. Then, the supplier sets $\omega$ and $g$ according to the retailer's decisions.

In the centralized GAPSC, the supplier and the retailer act as an entity aiming to maximize the total supply chain profit. The optimization problem of the centralized GAPSC can be obtained by summing up Eqs. (1) and (2):

$$
\max _{p, g, S} \Pi(p, g, S)=(p-c-\rho g)(\alpha-\beta p+\gamma g+\delta S)-\frac{\mu}{2} g^{2}-\frac{\lambda}{2} S^{2}
$$

Assumption 1 To ensure the various profit functions have a unique optimum, $\mu\left(2 \beta \lambda-\delta^{2}\right) \geq \lambda(\gamma-\beta \rho)^{2}$ is assumed.

The optimal equilibriums and profits of GAPSC are given in Table 1 and Table 2.

\section{Analysis}

In this section, the optimal decisions of GAPSC are compared to study the influence of channel power structure on the supply chain.

Theorem 1 Compared with the centralized GAPSC, the green level of the agricultural products, shelf space allocated and the total profits in the decentralized GAPSC are lower.

Theorem 1 shows that centralized GAPSC not only gains the greatest profits, but also supplies the agricultural products with the highest green level and allocates the most shelf space. It shows that the performances of the decentralized GAPSCs are relatively low.

Theorem 2 In the decentralized GAPSC, the shelf space allocated, green level of agricultural products and sales quantity satisfy the following order. 
(1) When $\gamma \geq \beta \rho$ and $\mu \delta^{2} \geq \lambda(\gamma-\beta \rho)^{2}, \quad S_{r}^{S^{*}} \geq S_{r}^{R^{*}}, g_{s}^{S^{*}} \geq g_{s}^{R^{*}}, \quad q_{s}^{S^{*}} \geq q_{s}^{R^{*}}$.

(2) When $\gamma \geq \beta \rho$ and $\mu \delta^{2} \leq \lambda(\gamma-\beta \rho)^{2}, S_{r}^{S^{*}} \leq S_{r}^{R^{*}}, g_{s}^{S^{*}} \leq g_{s}^{R^{*}}, q_{s}^{S^{*}} \leq q_{s}^{R^{*}}$.

(3) When $\gamma \leq \beta \rho, \quad S_{r}^{S^{*}} \geq S_{r}^{R^{*}}, \quad g_{s}^{S^{*}}=g_{s}^{R^{*}}=0, \quad q_{r}^{S^{*}} \geq q_{r}^{R^{*}}$.

Theorem 2 shows that when the supplier has incentives to make some effort in R\&D of green technology and the unit R\&D cost is high enough, the sales quantity, green level and shelf space allocated in the supplier-Stackelberg GAPSC are higher. Otherwise, the sales quantity, green level and shelf space allocated in the retailer-Stackelberg GAPSC are much higher. When the supplier has no incentives to make some effort in R\&D of green technology, the sales quantity and shelf space allocated in the supplier-Stackelberg GAPSC are always higher than that in the retailer-Stackelberg GAPSC.

Table 1 Optimal equilibriums of GAPSC

\begin{tabular}{|c|c|c|c|c|}
\hline & \multirow{2}{*}{ Centralized GAPSC } & \multicolumn{2}{|c|}{ Decentralized GAPSC } \\
\hline & & & Supplier-Stackelberg GAPSC & Retailer-Stackelberg GAPSC \\
\hline \multirow{5}{*}{$\gamma \geq \beta \rho$} & $\omega^{*}$ & - & $\frac{\left[\mu\left(2 \beta \lambda-\delta^{2}\right)+\lambda \beta \rho(\gamma-\beta \rho)\right](\alpha-\beta c)}{\beta\left[2 \mu\left(2 \beta \lambda-\delta^{2}\right)-\lambda(\gamma-\beta \rho)^{2}\right]}+c$ & $\frac{\lambda[\mu+\rho(\gamma-\beta \rho)](\alpha-\beta c)}{\mu\left(4 \beta \lambda-\delta^{2}\right)-2 \lambda(\gamma-\beta \rho)^{2}}+c$ \\
\hline & $p^{*}$ & $\frac{\lambda[\mu+\rho(\gamma-\beta \rho)](\alpha-\beta c)}{\mu\left(2 \beta \lambda-\delta^{2}\right)-\lambda(\gamma-\beta \rho)^{2}}+c$ & $\frac{\left[\mu\left(3 \beta \lambda-\delta^{2}\right)+\beta \lambda \rho(\gamma-\beta \rho)\right](\alpha-\beta c)}{\beta\left[2 \mu\left(2 \beta \lambda-\delta^{2}\right)-\lambda(\gamma-\beta \rho)^{2}\right]}+c$ & $\frac{\lambda[3 \beta \mu+(2 \beta \rho-\gamma)(\gamma-\beta \rho)](\alpha-\beta c)}{\beta\left[\mu\left(4 \beta \lambda-\delta^{2}\right)-2 \lambda(\gamma-\beta \rho)^{2}\right]}+c$ \\
\hline & $g^{*}$ & $\frac{\lambda(\gamma-\beta \rho)(\alpha-\beta c)}{\mu\left(2 \beta \lambda-\delta^{2}\right)-\lambda(\gamma-\beta \rho)^{2}}$ & $\frac{\lambda(\gamma-\beta \rho)(\alpha-\beta c)}{2 \mu\left(2 \beta \lambda-\delta^{2}\right)-\lambda(\gamma-\beta \rho)^{2}}$ & $\frac{\lambda(\gamma-\beta \rho)(\alpha-\beta c)}{\mu\left(4 \beta \lambda-\delta^{2}\right)-2 \lambda(\gamma-\beta \rho)^{2}}$ \\
\hline & $S^{*}$ & $\frac{\delta \mu(\alpha-\beta c)}{\mu\left(2 \beta \lambda-\delta^{2}\right)-\lambda(\gamma-\beta \rho)^{2}}$ & $\frac{\delta \mu(\alpha-\beta c)}{2 \mu\left(2 \beta \lambda-\delta^{2}\right)-\lambda(\gamma-\beta \rho)^{2}}$ & $\frac{\delta \mu(\alpha-\beta c)}{\mu\left(4 \beta \lambda-\delta^{2}\right)-2 \lambda(\gamma-\beta \rho)^{2}}$ \\
\hline & $q^{*}$ & $\frac{\beta \lambda \mu(\alpha-\beta c)}{\mu\left(2 \beta \lambda-\delta^{2}\right)-\lambda(\gamma-\beta \rho)^{2}}$ & $\frac{\beta \lambda \mu(\alpha-\beta c)}{2 \mu\left(2 \beta \lambda-\delta^{2}\right)-\lambda(\gamma-\beta \rho)^{2}}$ & $\frac{\beta \lambda \mu(\alpha-\beta c)}{\mu\left(4 \beta \lambda-\delta^{2}\right)-2 \lambda(\gamma-\beta \rho)^{2}}$ \\
\hline \multirow{5}{*}{$\gamma \leq \beta \rho$} & $\omega^{*}$ & - & $\frac{\alpha+\beta c}{2 \beta}$ & $\frac{\lambda \alpha+\left(3 \beta \lambda-\delta^{2}\right) c}{4 \beta \lambda-\delta^{2}}$ \\
\hline & $p^{*}$ & $\frac{\lambda \alpha+\left(\beta \lambda-\delta^{2}\right) c}{2 \beta \lambda-\delta^{2}}$ & $\frac{\left(3 \beta \lambda-\delta^{2}\right) \alpha+\beta\left(\beta \lambda-\delta^{2}\right) c}{2 \beta\left(2 \beta \lambda-\delta^{2}\right)}$ & $\frac{3 \lambda \alpha+\left(\beta \lambda-\delta^{2}\right) c}{4 \beta \lambda-\delta^{2}}$ \\
\hline & $g^{*}$ & 0 & 0 & 0 \\
\hline & $S^{*}$ & $\frac{\delta(\alpha-\beta c)}{2 \beta \lambda-\delta^{2}}$ & $\frac{\delta(\alpha-\beta c)}{2\left(2 \beta \lambda-\delta^{2}\right)}$ & $\frac{\delta(\alpha-\beta c)}{4 \beta \lambda-\delta^{2}}$ \\
\hline & $q^{*}$ & $\frac{\beta \lambda(\alpha-\beta c)}{2 \beta \lambda-\delta^{2}}$ & $\frac{\beta \lambda(\alpha-\beta c)}{2\left(2 \beta \lambda-\delta^{2}\right)}$ & $\frac{\beta \lambda(\alpha-\beta c)}{4 \beta \lambda-\delta^{2}}$ \\
\hline
\end{tabular}

Table 2 Optimal profits of GAPSC

\begin{tabular}{|c|c|c|c|c|}
\hline & \multirow{2}{*}{ Centralized GAPSC } & \multicolumn{2}{|c|}{ Decentralized GAPSC } \\
\hline & & & Supplier-Stackelberg GAPSC & Retailer-Stackelberg GAPSC \\
\hline \multirow{6}{*}{$\gamma \geq \beta \rho$} & $\pi_{s}^{*}$ & - & $\lambda \mu(\alpha-\beta c)^{2}$ & $\mu \lambda^{2}\left[2 \beta \mu-(\gamma-\beta \rho)^{2}\right](\alpha-\beta c)^{2}$ \\
\hline & & & $2\left[2 \mu\left(2 \beta \lambda-\delta^{2}\right)-\lambda(\gamma-\beta \rho)^{2}\right]$ & $2\left[\mu\left(4 \beta \lambda-\delta^{2}\right)-2 \lambda(\gamma-\beta \rho)^{2}\right]^{2}$ \\
\hline & $\pi_{r}^{*}$ & - & $\lambda \mu^{2}\left(2 \beta \lambda-\delta^{2}\right)(\alpha-\beta c)^{2}$ & $\lambda \mu(\alpha-\beta c)^{2}$ \\
\hline & & & $2\left[2 \mu\left(2 \beta \lambda-\delta^{2}\right)-\lambda(\gamma-\beta \rho)^{2}\right]^{2}$ & $2\left[\mu\left(4 \beta \lambda-\delta^{2}\right)-2 \lambda(\gamma-\beta \rho)^{2}\right]$ \\
\hline & $\Pi^{*}$ & $\lambda \mu(\alpha-\beta c)^{2}$ & $\lambda \mu\left[3 \mu\left(2 \beta \lambda-\delta^{2}\right)-\lambda(\gamma-\beta \rho)^{2}\right](\alpha-\beta c)^{2}$ & $\lambda \mu\left[\mu\left(6 \beta \lambda-\delta^{2}\right)-3 \lambda(\gamma-\beta \rho)^{2}\right](\alpha-\beta c)^{2}$ \\
\hline & & $2\left[\mu\left(2 \beta \lambda-\delta^{2}\right)-\lambda(\gamma-\beta \rho)^{2}\right]$ & $2\left[2 \mu\left(2 \beta \lambda-\delta^{2}\right)-\lambda(\gamma-\beta \rho)^{2}\right.$ & $2\left[\mu\left(4 \beta \lambda-\delta^{2}\right)-2 \lambda(\gamma-\beta \rho)^{2}\right]^{2}$ \\
\hline \multirow{6}{*}{$\gamma \leq \beta p$} & $\pi_{s}^{*}$ & - & $\lambda(\alpha-\beta c)^{2}$ & $\beta \lambda^{2}(\alpha-\beta c)^{2}$ \\
\hline & & & $4\left(2 \beta \lambda-\delta^{2}\right)$ & $\left(4 \beta \lambda-\delta^{2}\right)^{2}$ \\
\hline & $\pi_{r}^{*}$ & - & $\lambda(\alpha-\beta c)^{2}$ & $\lambda(\alpha-\beta c)^{2}$ \\
\hline & & & $\overline{8\left(2 \beta \lambda-\delta^{2}\right)}$ & $\overline{2\left(4 \beta \lambda-\delta^{2}\right)}$ \\
\hline & $\Pi^{*}$ & $\lambda(\alpha-\beta c)^{2}$ & $3 \lambda(\alpha-\beta c)^{2}$ & $\lambda\left(6 \beta \lambda-\delta^{2}\right)(\alpha-\beta c)^{2}$ \\
\hline & & $2\left(2 \beta \lambda-\delta^{2}\right)$ & $8\left(2 \beta \lambda-\delta^{2}\right)$ & $2\left(4 \beta \lambda-\delta^{2}\right)^{2}$ \\
\hline
\end{tabular}

\section{Summary}

This paper studied a GAPSC composed of one supplier and one retailer in which the retail 
demand is sensitive to the green level and shelf space allocated. The optimal decisions are investigated by establishing game models of centralized GAPSC and decentralized GAPSC.

The contribution of this paper is twofold. Firstly, when will the supplier have incentives to make some effort in R\&D of green technology is analyzed. The results show that whatever the channel power structure is, only when the consumers are sensitive to the green level and insensitive to the retail price, the marginal cost related to the improvement of green level increases slowly, the supplier has incentives to make some effort in R\&D of green technology. Secondly, by calculating equilibrium results in the centralized and decentralized GAPSCs, the influence of different channel power structures on optimal decisions is analyzed. When the supplier makes some effort in R\&D of green technology and the unit R\&D cost of green technology is high enough, the sales quantity, green level and shelf space allocated in the supplier-Stackelberg GAPSC are much higher. When the supplier makes no effort in R\&D of green technology, the sales quantity and shelf space allocated in the supplier-Stackelberg GAPSC are always higher than that in the retailer-Stackelberg GAPSC.

Acknowledgement: The authors gratefully acknowledge the support from the National Science Foundation of China through grants 71402084, Shandong Province Soft Science Research Plan Project 2017RKB01374 and Humanities and Social Sciences Project of Shandong Provincial Education Department J14WG09.

\section{References}

[1] Ning C, Gao P, Wang B, et.al. Impacts of chemical fertilizer reduction and organic amendments supplementation on soil nutrient, enzyme activity and heavy metal content[J]. Journal of Integrative Agriculture 2017, 16(8): 1819-1831.

[2] Eisend M. Shelf space elasticity: A meta-analysis[J]. Journal of Retailing, 2014, 90(2): 168-181.

[3] Lu Q, Zhu D. Strategies of quality improvement in supply chains based on shelf space allocation[J]. Journal of Management Sciences in China, 2010, 13(1): 31-38.

[4] Luo X, Jiang Y, Hu Q. Supply chain coordination with shelf-space and retail price dependent demand and heterogeneous retailers[J]. Naval Research Logistics, 2010, 57(8): 673-685.

[5] Zhou Y, Zhong Y, Li J. An uncooperative order model for items with trade credit, inventory-dependent demand and limited displayed-shelf space[J]. European Journal of Operational Research, 2012, 223:76-85.

[6] Tsao Y, Lu J, An N, et.al. Retailer shelf-space management with trade allowance: A Stackelberg game between retailer and manufacturers[J]. International Journal of Production Economics, 2014, 148: 133-144.

[7] Amita R, Mehta P, Tripathi R. Optimal shelf-space stocking policy using stochastic dominance under supply-driven demand uncertainty[J]. European Journal of Operational Research, 2015, 246(1): 339-342.

[8] Hübner A, Schaal K. A shelf-space optimization model when demand is stochastic and space-elastic[J]. Omega, 2017, 68: 139-154.

[9] Ghosh, D, Shah J. A comparative analysis of greening policies across supply chain structures[J]. International Journal of Production Economics, 2012, 135 (2): 568-583.

[10] Huang Y, Wang K, Zhang T, et.al. Green supply chain coordination with greenhouse gases emissions management: a game-theoretic approach[J]. Journal of Cleaner Production, 2016, 112: 2004-2014.

[11] Jamali M, Rasti-Barzoki M. A game theoretic approach for green and non-green product pricing in chain-to-chain competitive sustainable and regular dual-channel supply chains[J]. Journal of Cleaner Production, 2018, 170: 1029-1043.

[12] Song H, Gao X. Green supply chain game model and analysis under revenue-sharing contract[J]. Journal of Cleaner Production, 2018, 170: 183-192.

[13] Banker R, Khosla I, Sinha K. Quality and competition[J]. Management Science, 1998, 44(9): 1179-1192. 